\title{
Instrumentos de Predicción
}

\author{
Dr.: Jairo Alberto Barragán Espinosa* \\ PRESENTADO' EN LA REUNION INTERREGIONAL OPS - OMS, SOBRE \\ TECNOLOGIA APROPIADA EN ATENCION PRENATAL \\ Washington. Diciembre 1984
}

\section{Los instrumentos de predicción dentro del marco de referencia que plantea "Salud para todos en el año 2000"}

Dentro de los planteamientos que se presentan en los documentos básicos de OMS y OPS para el desarrollo de estrategias hacia el cumplimiento de la meta de "Salud para todos en el año 2000", encontramos enunciados que hacen énfasis en la equidad, eficiencia y eficacia de los sistemas de salud. Estas condiciones, en la actualidad, por múltiples factores no se presentan en los países de América Latina, entre los cuales podemos observar: a) la dificultad de acceso de grandes grupos de población a los servicios de salud; b) rápido crecimiento de la demanda; c) deficiencias en el contenido, estructura y formas de operación de los servicios; d) problemas de índole financiero, que se au-

* Instructor Facultad de Medicina, Departamento de Ginecología y Obstetricia, Pontificia Universid ad Javeriana. mentan año por año, consistentes con la situación de franca recesión (1).

Lo anterior se traduce en bajas coberturas, baja productividad de los recursos, inequidad en el acceso real y utilización de los servicios por grupos altamente vulnerables (1).

Surge como estrategia fundamental para resolver estos limitantes, la Atención Primaria, "que es la estrategia que afecta y comprende a todo el sistema de salud y a toda la población que ese sistema supone servir".

La atención primaria propone resolver los problemas mediante una manera determinada de reorganizar y reorientar todos los recursos del sector salud para satisfacer las necesidades y aspiraciones en salud de una sociedad. De otro lado, y a manera de apoyo logístico, se presenta la comprobación empírica de que los problemas de salud de menor complejidad tienen mayor frecuencia relativa que 
los más complejos. De ahí la posibilidad de satisfacer los niveles de atención. En esta forma, la atención primaria implica la organización de servicios con base en niveles de atención dispuestos en una red de oferta previamente establecida $y$ cuya estructura y función se desea adaptar para obtener un proceso de cambio hacia el logro de "Salud para todos en el año 2000" (2).

La implantación de los niveles de atención crea la necesidad de hablar en términos de escalones de complejidad, que darán la organización interna de los niveles; supone por supuesto, la articulación de los diferentes niveles, para asegurar continuidad en la atención, por intermedio de un "sistema de referencia" que garantice la máxima eficiencia de los recursos (2).

De lo expuesto anteriormente, se deduce, que existiendo oferta de acuerdo con niveles de atención con escalas de complejidad se debe pensar en la estrategia de seleccionar los grupos de población que deberán ser atendidos en cada uno de ellos; se desprende una serie de conceptos como el de grupos humanos postergados, que se definen como aquellos grupos situados en condiciones de desigualdad, que están expuestos a varios factores de riesgo que afectan sus niveles de salud y bienestar; por lo tanto, la oferta deberá condicionarse para la adecuada atención de estos grupos brindándoles en cantidad y calidad los recursos óp timos para disminuir esa desigualdad (2).

Llegamos pues a tocar el concepto de factor de riesgo, elemento básico que va a permitirnos la identificación de los grupos postergados, y que trasciende la simple connotación biológica, orientada hacia conductas clínicas: así, un primer uso del concepto, es la identificación de una gama de condicionantes socioculturales, económicos y ambientales que lo integran. Deben tener los factores de riesgo, características de especificidad tan concretas que permitan orientar acciones eficaces y eficientes para su control.

\section{Proceso Metodológico de Elaboración de los Instrumentos de Predicción}

Al hablar de factores de riesgo, debemos aclarar algunos conceptos, sobre cómo los podemos encontrar. Siguiendo la metodología epidemiológica que plantea la OPS/OMS en su "Manual de Metodología de Enfoque de Riesgo" (2), debemos tener en cuenta los siguientes puntos:

a) Los factores de riesgo deben ser considerados como probabilidades que presenta un grupo o individuo de tener un daño determinado; de allí se desprende la necesidad de identificar el daño para poder investigar los factores de riesgo específicos que condicionan ese daño y no otro. En otras palabras es necesario "apellidar" el factor de riesgo.

b) Para efectos operativos: antes de entrar en la investigación de los factores de riesgo, es importante definir los problemas o "daños" prioritarios que afectan la situación de salud de un grupo; no es posible atacar todos los "daños" en forma simultánea y por ello se hace necesario priorizarlos, investigar sus correspondientes factores de riesgo y planear las estrategias para reducirlos.

c) Una vez encontrados los factores de riesgo que "posiblemente" predicen o condicionan un daño, es necesario entrar a valorarlos; desde el punto de vista de si su frecuencia es alta o baja en el grupo que estudiamos y si su 
frecuencia condiciona una probabilidad mayor o menor de sufrir el daño.

d) Con los factores de riesgo, ya valorados tanto en su prevalencia, como en su importancia frente al daño, estadística y causal, podemos elaborar un listado de los mismos e irlos ponderando con el fin de establecer un instrumento que al aplicarlo a los componentes del grupo, los clasificará en subgrupos de mayor o menor probabilidad de sufrir el daño; a ellos se les apellidará de al to o bajo riesgo de sufrirlo; de esta manera intentaremos predecir con la suficiente anticipación la ocurrencia de un daño y tendremos la oportunidad de reducirlo, suprimiendo o atenuando el efecto de los factores.

e) Entraremos luego a realizar la evaluación de ese instrumento, o sea a probar si esos factores de riesgo predecían en forma confiable el resultado; realizaremos pruebas que tienen que ver con el poder predictivo positivo o negativo, la sensibilidad y la especificidad. De acuerdo con lo anterior lo ajustaremos convenientemente.

f.) Finalmente usaremos ese listado de factores, como instrumento de predicción. para detectar la probabilidad de ocurrencia de un daño, realizar acciones tendientes a suprimir los factores de riesgo y en resumen a disminuir la presencia del daño, obteniendo una mejor calidad de vida del grupo vulnerable.

3. Beneficios del empleo de los instrumentos de predicción en la prestación de servicios

Además de categorizar los grupos de acuerdo con los grados de riesgo que pre- sentan, el empleo de los instrumentos de predicción en la atención de salud con enfoque de Riesgo, permite:

a) Asumir un criterio preventivo en la atención.

b) Desarrollar adecuadamente los niveles de atención de acuerdo con escalones de complejidad.

c) Racionalizar los recursos de todo tipo dando la oportunidad de ofrecer mejores servicios a quienes más lo necesitan $y$,

d) Conocer, de acuerdo con el instrumento, las proporciones reales de la población clasificada según grado de riesgo, y de esta manera, reorientar los recursos para lograr un aumento de coberturas de la atención para cada uno de los riesgos.

En resumen, permite mejorar la efectividad de la atención aplicando un enfoque preventivo que da lugar a actuar con oportunidad sobre la disminución de los daños.

\section{Experiencia en Colombia}

La Federación Panamericana de Facultades y Escuelas de Medicina FEPAFEM, y la Asociación Colombiana de Facultades de Medicina ASCOFAME, de común acurerdo iniciaron en 1969 la aplicación en Colombia del concepto de Riesgo Materno Fetal con base en la experiencia del Dr. Roberto Nesbitt adelantada en Estados Unidos.

Se inició con el diseño de un formulario que consideró diversos factores que tenían incidencia previsible en el resultado del parto, en lo que respecta a la madre $y$ al recién nacido. Con base en un riguroso análisis médico se asignaron pesos a cada uno de los factores considerados, 
que sumados, producían en conjunto el índice de riesgo que puede conllevar un embarazo.

En forma similar se examinó un formulario del resultado final del parto y se asignaron pesos numéricos a las diferentes circunstancias indicativas de un resultado de riesgo.

Los formularios diseñados fueron aplicados en servicios de atención médica adscritos a la Universidad Javeriana de Bogotá (Hospital San Ignacio y Centros de Salud).

Al cabo de dos años se recolectaron un total de 664 casos completos (Indice de riesgo y resultado del parto) que sirvieran para realizar el primer estudio con el fin de analizar la consistencia interna, predictibilidad y confiabilidad del instrumento.

Para tal estudio se utilizaron técnicas estadísticas como correlación múltiple, interacción de variables y análisis de clasificación múltiple.

Los resultados obtenidos mostraron que el índice utilizado tenía buena capacidad predictiva y señalaron aquellos aspectos susceptibles de modificaciones para hacerlo más eficiente.

Por lo tanto se rediseñaron los formularios suprimiendo algunas variables que no incidían en el resultado y cambiando algunos pesos dados inicialmente; además se estableció una doble clasificación de riesgo; la inicial que nos permitía conocer el riesgo en el embarazo y la final al octavo mes de gestación, que daba la clasificación para el parto y al mismo tiempo permitía conocer la eficacia del tratamiento efectuado.
Teniendo como punto de partida esta experiencia inicial, el enfoque de riesgo en Colombia tuvo dos aplicaciones; la primera en el Programa de Atención Materno Infantil -PAMI- de la Universidad Javeriana, cuyo propósito era el de estructurar un modelo que permitiera Atención Integral de las pacientes en los aspectos preventivos y asistenciales, logro de altas coberturas de atención de la población más vulnerable, reasignación de funciones del personal de salud, participación de la comunidad en la organización y funcionamiento de los servicios de salud, regionalización de los servicios y evaluación sistemática de cada uno de los aspectos y con los siguientes objetivos:

- Investigativo: El estudiante fue adquiriendo interés en el manejo del instrumento de clasificación que permitía orientar conductas durante el embarazo y el parto.

- Docente: La enseñanza de la GinecoObstetricia y la Pediatría se hicieron cada vez más extramurales y permitieron al estudiante ponerse en contacto con la paciente, su familia y sus condiciones ambientales.

- Prestación de los servicios: Se organizaron manejos técnicos y sistemas de remisión de pacientes de los Centros periféricos al Hospital Universitario.

EI PAMI realizó con 1.015 casos una segunda evaluación de los formularios en 1978, con el fin de perfeccionarlos. Se utilizó el análisis discriminante y de correlación; con esta evaluación el formulario de riesgo se simplificó un poco pero el poder predictivo aumentó al doble y además se eliminaron algunas inconsistencias internas existentes.

Actualmente este formulario se utiliza en el Programa con muy buenos resul- 
tados, tanto a nivel asistencial como docente.

La segunda aplicación de enfoque de riesgo fue realizada por el Ministerio de Salud de Colombia motivada por la reorganización del Sistema Nacional de Salud del País.

Esta reorganización implicaba un esquema piramidal que tenía como bases, niveles de atención de los pacientes de acuerdo con su patología y mecanismos de remisión para control y seguimiento.

Por otra parte, y como estrategia para lograr aumento en las coberturas se comenzó la asignación de funciones en personal médico, de enfermería y voluntario de la comunidad (promotoras, parteras emp íricas).

Esta coyuntura organizacional dio pie para favorecer la implantación del modelo de Riesgo Materno Infantil que ha facilitado:

- El desarrollo de organización administrativa de la Regionalización.

- Atención de pacientes por niveles.

- Asignación específica de funciones y actividades por personas que trabajan en los diferentes niveles.

- Vinculación de personal de la comunidad en la atención obstétrica (parteras empíricas).

- Mayor y mejor conocimiento de la comunidad, sobre su situación de salud y de la atención en el nivel adecuado de acuerdo con la necesidad.

La organización de los niveles de atención según la clasificación por el riesgo materno fetal es la siguiente:
Bajo Riesgo: Para ofrecer atención a las embarazadas clasificadas en Riesgo Bajo, que no presentan ninguna patología y sus condiciones socio-económicas y de fecundidad son buenas, se ha previsto en el Ministerio de Salud, ofrecer un nivel "primario de atención". Este nivel involucra hospitales de tipo local, centros y puestos de salud y atención a domicilio por personal de comunidad con previa capacitación. Quiere esto significar que el equipo humano que maneja este nivel de riesgo está conformado por Médico General, Enfermera, Auxiliar de Enfermería, Promotora de Salud y Partera Empírica complementada.

La dotación es la mínima necesaria, para poder dar atención institucional a nivel domiciliario.

Este tipo de organización se ha estado desarrollando en forma progresiva a medida que el personal se ha capacitado, y las condiciones de infraestructura lo han permitido.

Riesgo Medio: En la misma forma que se organizó la atención en el "nivel primario", se ha desarrollado un nivel secundario que implica un manejo técnico instrumental más complejo.

Por tanto, el nivel de atención se ha asignado en Hospitales locales de buen desarrollo y Hospitales de tipo regional.

Los funcionarios encargados de manejar a las pacientes con este Riesgo, son fundamentalmente el Médico General bien capacitado y en ocasiones el GinecoObstetra. Este nivel permite un manejo más especializado, tanto para el diagnóstico como para el tratamiento y seguimiento, requiriéndose equipos de laboratorio, quirúrgicos y de monitoría mínimos, paŕa obtener la calidad que amerita el 
grupo de obstétricas clasificadas en este Riesgo.

Para lograr el desarrollo de este nivel, hemos encontrado más dificultades que para el anterior por las limitantes en dotación y recurso humano calificado, sin embargo a medida que se ha podido concientizar al equipo de salud y los presupuestos lo han permitido, se ha logrado una depuración en la atención cada vez mayor.

Riesgo Alto: Finalmente, con el propósito de ofrecer una al ta calidad de atención Gineco-obstétrica en el país, que responda a las necesidades de las pacientes de Alto Riesgo, se ha normado establecer un nivel de Atención Terciaria.

Este nivel estaría conformado por Hospitales Regionales de buen desarrollo y Hospitales Universitarios o especializados que tengan o vayan adquiriendo elementos de alta tecnología (ecógrafos, monitores, laboratorio de alto riesgo para estudios de líquido amniótico y otros)

El recurso humano será el de mayor calificación, bien sea que exista o que se vaya capacitando.

Este nivel exige no sólo la actividad de atención clínica-quirúrgica, sino que necesita organizar y fomentar una coordinación permanente y efectiva y un sistema de auditoría y educación continua para los niveles inferiores. El plan se ha venido desarrollando en forma coordinada con los hospitales universitarios, la Federación Colombiana de Sociedades de Ginecología y Obstetricia, FECOLSOG, $y$ algunos hospitales de tipo regional.

\section{a) Evaluación del Instrumento de Ries- go por el Ministerio de Salud}

De las 1.998 pacientes obstétricas, las clasificadas en Riesgo Bajo contribuyen con el mayor volumen del total de embarazadas, es así como el $74.6 \%$ al iniciar el control prenatal quedaron ubicadas en este Riesgo. Al control en el 9o. mes permanecen el $94.5 \%$ en el mismo Riesgo Bajo, elevan el $0.3 \%$ a medio y el $1.5 \%$ a al to. No regresaron a control el $3.7 \%$.

El volumen de embarazadas clasificadas en Riesgo Medio al inicio de su gestación fue de 456 casos que corresponde al 22.9\%; de los cuales al 80 . mes continuaron en el mismo riesgo el $96 \%$, pasaron a Riesgo Alto el $2.0 \%$ y descendieron a Riesgo Bajo el $1.1 \%$.

Al entrar en el análisis del Riesgo Alto, encontramos que 49 mujeres iniciaron el embarazo en este Riesgo que representan el $2.5 \%$ del total de embarazadas del estudio. De estas embarazadas, 40 llegan en su mismo riesgo al 80. mes $(81.6 \%)$. Las 9 restantes se distribuyeron: 1 en Riesgo Medio $(2.0 \%)$ y 5 en Riesgo Bajo (10.2\%), las 3 pacientes restantes se perdieron del estudio en este lapso $(6.1 \%)$.

El volumen total de pacientes de Alto Riesgo en el 8o. mes aumenta con pacientes preferentemente provenientes de Riesgo Bajo.

Contrastando el índice de Riesgo con el Resultado Final del parto se observa una predicción bastante buena ya que el $98.8 \%$ de los casos que tuvieron un resultado normal (Bajo) estaban clasificados como de Riesgo Bajo, el $94.9 \%$ de los que tuvieron algunas complicaciones (Medio) estaban clasificados como de Riesgo Medio y el $95 \%$ de los que tuvieron en el parto complicaciones graves (Alto) venían clasificados como de Riesgo Alto.

Conviene destacar que el hecho peligroso de clasificar una mujer en riesgo 
bajo y su parto sea con muchas complicaciones (Alto), en el presente estudio sólo sucedió en dos casos.

\section{b) Proyecciones en la Aplicación de Riesgo en Colombia}

Somos conscientes de que a pesar de los logros positivos que hemos obtenido, estos constituyen sólo el inicio del proceso aplicativo del Modelo de Riesgo, pues existen una serie de proyecciones en su utilización, entre las cuales podemos enumerar:

- Diversificación del Modelo de Clasificación de Riesgo.

- Organización de Historia Clínica Modular por niveles de Atención con base en Riesgo.

- Definición y elaboración de técnicas $y$ procedimientos unificados en obstetricia.

- Proceso racionalizado en la administración de recursos.

- Ajustes curriculares en la enseñanza del área de la salud.

\section{Diversificación del Modelo de Clasifica- ción de Riesgo}

En la actualidad, se ha llegado a un Modelo único simplificado que ha mostrado su efectividad, sin embargo, el ser aplicado en un país como Colombia, que tiene zonas urbanas, rurales y áreas geopolíticas disímiles, las cuales crean o condicionan factores de riesgo diferentes, se hacen necesarios los ajustes de algunas variables y la inclusión de otras, por áreas y por zonas, para darle mayor aplicabilidad y sensibilidad.
A medida que se logren completar un número de "casos", estadísticamente significativos y luego del procesamiento y análisis discriminante, se irán produciendo los ajustes regionales.

\section{Organización de Historia Clínica Modular por Niveles de Atención con base en Riesgo}

Los esquemas de clasificación de Riesgo, actualmente se han venido utilizando como indicadores para definir niveles de atención y organización de pacientes al nivel correspondiente; sinembargo se considera que el actual esquema puede y debe ser utilizado como parte de la historia clínica básica precodificada, que complementa los módulos a medida que se asciende en el nivel de atención.

Cabe anotar que este proceso está en curso y los indicadores de Riesgo han quedado incluidos en la Historia Clínica básica para el país, normada por el Ministerio de Salud. Por otra parte, conjuntamente con la Federación Colombiana de Sociedades de Gineco-Obstetricia (FECOLSOG) se está adelantando el Módulo de Atención Perinatal para el manejo del Riesgo Alto que complementa la Historia Clínica Básica.

\section{Defínición y Elaboración de Técnicas y Procedimientos Unificados en Obstetricia}

Habiendo definido las funciones para cada nivel y funcionario del Sistema $\mathrm{Na}$ cional de Salud de acuerdo con la clasificación de riesgo, se ha iniciado la elaboración de Manuales de Técnicas y Procedimientos, con el propósito de unificar el manejo de pacientes y evitar la continuidad del sistema anárquico e individualista de la atención que lleva una dispersión de los recursos. 
Los Manuales de Técnicas y Procedimientos para el manejo obstétrico en el país, se están diseñando en forma de guías de consulta para cada funcionario que tiene responsabilidades asignadas. Es así como se tendrá:

- Manual para el especialista (GinecoObstetra).

- Manual para el Médico General.

- Manual para la Enfermera y Auxiliar de Enfermería.

- Manual para la Promotora de Salud.

- Manual para la Partera Empírica Complementada.

\section{Proceso Racionalizado en la Administra- ción de Recursos}

Una vez definidos y normados el manejo de las pacientes, a través de las funciones y los Manuales de Técnicas y Procedimientos, se producirán los ajustes para adecuar los recursos a la necesidad de los niveles y las personas que van a ofrecer servicios de salud.

Esto implica, por ejemplo: que al definirle exactamente a la partera empírica "el por qué", "el dónde", "el cuándo" y "el cómo" va a realizar el control de embarazo de Riesgo Bajo asignado, se le tendrá que entregar "el con qué" en el orden de: conocimiento, elementos de dotación, drogas, papelería y demás implementos que se consideren necesarios. En esta forma se llegará hasta el nivel más especializado, donde se atenderá el Riesgo Alto con los Recursos más calificados y sofisticados.

Este proceso favorecerá la máxima utilización de los recursos y evitará la do- tación ińadecuada y sub-utilizada como se aprecia con mucha frecuencia. Esta etapa no es fácil, porque cada institución por pequeña que sea, quiere recursos humanos y dotación del más alto nivel para aumentar su prestigio; por otra parte $y$ en múltiples oportunidades existen otros sectores extraños al de la salud, que intervienen en este aspecto.

Sin embargo, este sistema racional es indispensable en un país económicamente débil, que no puede darse el lujo de despilfarrar sus deficientes recursos económi$\cos y$ que está tratando de organizar niveles de atención de acuerdo con Riesgo y demanda organizada de los servicios por parte de la comunidad.

Toda esta adecuación de recursos, se traducirá en servicios que serán evaluados tanto en cantidad como en calidad, a través de indicadores desarrollados para tal fin, que permitan hacer los ajustes permanentes en cualquier área.

\section{Ajustes Curriculares en la Enseñanza del Area de la Salud}

En la medida que se logre obtener avances positivos altamente confiables $y$ evaluados en las Instituciones que ofrecen los Servicios Coordinadamente con las Entidades docentes y asociaciones científicas, se promoverán modificaciones en la enseñanza de la Gineco-obstetricia, tratando de ajustar los contenidos docentes a las exigencias que va a tener el médico general y el personal de enfermería para atender pacientes de Nivel Local (Riesgo Medio y Bajo).

También se promoverá la unificación de la enseñanza en Técnicas y Procedimientos, teniendo en cuenta los Manuales producidos entre FECOLSOG y el Mi- 
nisterio de Salud. Es obvio que este material servirá de guía y no pretende reemplazar los textos.

Otra actividad que se proyecta trabajar es la coordinación y cooperación del equipo de salud que va a brindar servicios en los distintos niveles. De esta integración va a depender en un porcentaje muy alto, la buena marcha de la asignación de funciones, la remisión y contra-remisión de pacientes y demás puntos de la organización de los servicios.

\section{RESUMEN}

La OPS/OMS, teniendo en cuenta la meta de "Salud para todos en el año 2000", ha planteado dentro de las estrategias fundamentales para lograrla la "Atención Primaria en Salud".

De acuerdo con la situación actual de salud de los países del tercer mundo, la atención primaria en salud entra a jugar un papel importante en la prestación de los servicios y con el fin de hacerlos relevantes, se consideran los conceptos de niveles de atención como escalones de complejidad para brindar una oferta de buena calidad a la población, de acuerdo con sus posibilidades de acceso o grado de postergación.

Para identificar aquellos grupos postergados debe incorporarse el concepto de Riesgo, que permite abordar la problemática de salud de un grupo determinado con un criterio preventivo, toda vez que conociéndose el o los factores de riesgo que inciden sobre la presencia de un daño, en un grupo o individuo, ofrece la posibilidad de intervenir con el fin de reducir o atenuar esos factores mucho antes de presentarse el resultado no deseado.
Los factores de riesgo que inciden en la mayor probabilidad de presencia del daño son el fundamento de un instrumento de predicción que, al aplicarlo, permitirá clasificar grupos o individuos según mayor o menor riesgo de enfermar o morir por causa de un daño específico.

La aplicación en Colombia del Enfoque de Riesgo, especialmente en el área materno infantil, data desde 1969; inicialmente tuvo connotación investigativa, con el fin de ajustar gradualmente los formularios en cuanto a sensibilidad y especificidad, lo que permitió que posteriormente se comenzara su utilización en el Programa de Atención Materno Infantil, PAMI de la Universidad Javeriana de Bogotá y en el Ministerio de Salud a nivel nacional.

De esa aplicación, se han obtenido resultados positivos que se detallan en el texto; así como se han detectado limitantes que inciden en forma importante en el desarrollo de la Atención Materno Infantil con el Enfoque de Riesgo.

Como consecuencia del proceso a nivel nacional, se tienen las siguientes proyecciones: a) de especificación de los instrumentos de predicción para diferentes regiones del país; b) de incorporación de los mismos a los Registros Médicos del Sistema Nacional de Salud, lo cual ya se está realizando; c) de definición y elaboración de normas y procedimientos de cada miembro del equipo de salud correspondiente $y$ de acuerdo con niveles de atención según los criterios del enfoque de riesgo; d) de mayor racionalización en el uso de los recursos según los niveles de atención, de acuerdo con su complejidad y por el tipo de riesgo que atienden.

Finalmente, y como conclusión se desprende que estos instrumentos de pre- 
dicción no son sólo un conjunto de factores de riesgo que permiten clasificar a un grupo, sino evidentemente un instrumento de cambio en las políticas de ofer-

\section{BIBLIOGRAFIA}

1. O. P. S., Salud Materno Infantil y Atención Primaria en las Américas. (Hechos y tendencias). Publicación Científica No. 461, 1984.

2. O. P. S., Manual de Metodología de Enfoque de Riesgo. Publicación en revisión. ta de servicios, que hacen más compatible la aplicación de las estrategias tendientes a dar "Salud para todos en el año 2000".

3. DAZA PARADA LUIS, Investigación de Riesgo Materno Infantil, Primer Documento. Minsalud - Universidad Javeriana Universidad del Valle 1976.

4. DAZA PARADA LUIS, BARRAGAN ESPINOSA JAIRO, Atención Obstétrica Recién Nacido y Riesgo Materno Infantil. Evaluación y Proyecciones, 1978-1981. Cuarto Documento, 1983. 